\title{
Effect of Different Adhesion Protocols on the Shear Bond Strength of Universal Adhesive Systems to Sound and Artificial Caries-Affected Dentin
}

\author{
Omnia M. Sami ${ }^{1}$ Essam A. Naguib ${ }^{1} \quad$ Rasha H. Afifi ${ }^{1}$ Shaymaa M. Nagi \\ ${ }^{1}$ Conservative Dentistry Department, Faculty of Oral and Dental \\ Medicine, Future University, Cairo, Egypt \\ ${ }^{2}$ Oral and Dental Research Division, Restorative and Dental \\ Materials Department, National Research Centre, Cairo, Egypt \\ Eur J Gen Dent 2021;10:30-36. \\ Address for correspondence Shaymaa M. Nagi, PhD, Oral and \\ Dental Research Division, Restorative and Dental Materials \\ Department, National Research Centre, 33 El Bohouth st. \\ (former El Tahrir st.), Dokki, Giza, P.O. 12622, Egypt \\ (e-mail: smnagi@gmail.com).
}

\begin{abstract}
Keywords

- caries-affected dentin

- microshear

- multimode

- universal dental adhesives

- adhesion protocols
\end{abstract}

Objective Bonding to different dentin substrates influences resin composite restoration outcomes. This study investigated the influence of different adhesion protocols on the shear bond strength of universal adhesive systems to sound and artificial caries-affected dentin (CAD).

Materials and Methods Occlusal enamel of 80 premolars were wet grinded to obtain flat midcoronal dentin. Specimens were equally divided according to the substrate condition: sound and CAD by subjecting to $\mathrm{pH}$-cycling for 14 days. Each dentin substrate was bonded with the adhesive systems used in this study: Single Bond universal adhesive or Prime\&Bond universal (applied either in etch-and-rinse or self-etch adhesion protocol). Adhesive systems were utilized according to manufacturers' instructions, then resin composite was built up. Specimens were tested for shear bond strength. The data were analyzed by three-way analysis of variance, and failure modes were determined using stereomicroscope.

Results There was no statistically significant difference between the two tested adhesive systems on artificially created CAD with different adhesion protocols. On sound dentin, Single Bond universal, either in the etch-and-rinse or self-etch adhesion protocols, revealed higher statistically significant shear bond strength mean values compared with CAD.

Conclusion Single Bond universal adhesive in an etch-and-rinse adhesion protocol improved only bonding to sound dentin, while no added positive effect for the etching step with Prime\&Bond universal adhesive was found when bonded to both sound and $C A D$ substrates. The influence of CAD on the performance of the universal adhesives was material-dependent.

\section{Introduction}

Bonding to dentin substrate still represents a challenge in clinical practice, as dentin has a nonuniform permeability, which is moisture; moreover, it is a dynamic structure. In restorative dentistry studies concerning bonding to normal dentin are still prevalent. Even though efforts regarding caries-affected dentin (CAD) have been seen recently,
DOI https://doi.org/ $10.1055 / \mathrm{s}-0041-1732776$ ISSN 2320-4753
(C) 2021. European Journal of General Dentistry.

This is an open access article published by Thieme under the terms of the Creative Commons Attribution-NonDerivative-NonCommercial-License, permitting copying and reproduction so long as the original work is given appropriate credit. Contents may not be used for commercial purposes, or adapted, remixed, transformed or built upon. (https://creativecommons.org/licenses/by-nc-nd/4.0/).

Thieme Medical and Scientific Publishers Pvt. Ltd. A-12, 2nd Floor, Sector 2, Noida-201301 UP, India 
selective caries elimination has been extensively supported to conserve the tooth substrate and prevent avoidable pulp tissue exposure. Thus, resin composite is bonded to the cavity floor that commonly comprises of CAD, after the elimination of infected dentin. It might assume that the structural and morphological alterations within CAD may negatively impact the performance of dental materials applied to it. ${ }^{1,2}$

There is still a controversy about the performance of the universal adhesive systems ("multimode" or "multipurpose" adhesives), ${ }^{3}$ which could be applied with different adhesion protocols (self-etch or etch-and-rinse) according to the clinical situation on the bond strength to CAD. Thus, the current study was conducted to assess the effect of bonding to CAD on the shear bond strength (SBS) of two universal adhesives applied in different adhesion protocols compared with sound dentin.

The null hypotheses that could be accepted or rejected were that: (1) there was no difference in resin-dentin SBS of CAD and sound dentin; (2) there was no difference in resin-dentin SBS between the two tested universal adhesive systems; and (3) there was no difference in resin-dentin SBS between the different adhesion protocols (either in etch-and-rinse or self-etch).

\section{Materials and Methods}

\section{Teeth Selection}

Eighty freshly extracted sound human upper premolars were selected from patients aged 18 to 25 years. Teeth were cleaned from periodontal fibers and soft tissues under running tap water using sharp hand scaler. Teeth were also examined for cracks, caries, fracture, or any pathological abnormalities using magnifying lens $(25 \times)$ and only teeth free from any defect were selected. Teeth were then kept at $4^{\circ} \mathrm{C}$ soaked in distilled water, for a maximum period of 3 months after extraction with a weekly change of the distilled water. ${ }^{4}$

\section{Grouping of Specimens}

The 80 selected premolars were divided into two main equal groups ( $n=40)$ according to the dentin substrate condition (A); either sound dentin $\left(A_{1}\right)$ or artificially CAD $\left(A_{2}\right)$. Each main group was divided into two equal subgroups $(n=20)$, according to the universal adhesive system utilized (B): Single Bond universal adhesive system $\left(B_{1}\right)$ and Prime\&Bond universal adhesive system $\left(B_{2}\right)$. Each subgroup was divided into two equal classes $(n=10)$ according to the adhesion protocol $(C)$ utilized: either etch-and-rinse $\left(C_{1}\right)$ adhesion protocol or self-etch $\left(\mathrm{C}_{2}\right)$ adhesion protocol.

\section{Specimens Preparation}

Roots of all teeth were cut $2 \mathrm{~mm}$ below cementoenamel junction by a double-sided diamond disc mounted to low-speed handpiece. The contents of the pulp chamber were then removed with a broach. Occlusal enamel was removed by wet grinding using a 240 -grit silicon carbide ( $\mathrm{SiC}$ ) abrasive paper mounted in a bench grinder machine to expose midcoronal dentin. Checking the remaining dentin thickness was done using precise caliper. Only specimens of remaining dentin thickness " $2 \mathrm{~mm}$ " were used in the current study. Each exposed dentin surface was finished using wet SiC 600-grit paper for 60 seconds in a circular motion to create a standardized smear layer. ${ }^{5}$

Each dentin specimen was embedded in the acrylic resin up to the cementoenamel junction, with the prepared occlusal surface facing outward. All specimens were immediately immersed in distilled water after complete acrylic resin polymerization until being used.

\section{Development of Caries-Affected Dentin (Artificial Caries)}

Half of the dentin specimens $(n=40)$ were exposed to cariogenic challenge by $\mathrm{pH}$ cycling to produce artificial caries lesion. $\mathrm{pH}$ cycling procedure and demineralizing and remineralizing solutions preparation were done according to Nicoloso et al. ${ }^{6}$

\section{Application of the Tested Universal Adhesive Systems with Different Adhesion Protocols on Both Sound and Caries-Affected Dentin Substrates}

For standardization of the bonded area, all dentin specimens were covered with an adhesive tab that provided a hole of $2 \mathrm{~mm}$ diameter, located at the center of dentin surface. Both tested universal adhesives were applied either in etch-and-rinse or self-etch adhesion protocols according to the assigned group. Materials name, manufacturer, chemical composition, and details of application steps are presented in - Table 1.

\section{Application of the Nanohybrid Resin Composite}

After bonding procedure has been completed, Filtek Z250 nanohybrid universal resin composite (3M ESPE, United States) was packed in a cylindrical transparent plastic tube of inner dimensions-2 $\mathrm{mm}$ diameter and $3 \mathrm{~mm}$ height-which act as a mold during resin composite application. ${ }^{7}$ Resin composite was packed in two increments of $1.5 \mathrm{~mm}$ each, then each increment was light cured for 10 seconds according to the manufacturer's instructions using LED light curing unit of $1,000 \mathrm{~mW} / \mathrm{cm}^{2}$. After removing the plastic tubes, all resin composite cylinders were checked using magnifying glass lens $(25 \times)$ to detect any defects. If any imperfection was found in any resin composite cylinder, the specimen was discarded. Each restored specimen was stored in $15 \mathrm{~mL}$ artificial saliva ( $\mathrm{pH}=7$ ) in an incubator at $37^{\circ} \mathrm{C}$ for 48 hours until testing. Artificial saliva composition was prepared according to Pashley et al. ${ }^{8}$

\section{Shear Bond Strength Test}

SBS test was performed for all bonded specimens using a universal testing machine (model LRX-plus; Lloyd Instruments Ltd; Fareham, United Kingdom). A chisel-shaped shearing blade with a $0.5 \mathrm{~mm}$ wide sharp edge was aligned parallel to the flat dentin surface of the bonded specimen. The load cell control system was then adjusted to apply load force of $5 \mathrm{~N}$ by chisel on dentin-restoration interface at a crosshead speed of $0.5 \mathrm{~mm} / \mathrm{min}$ until failure occurs. The control system and its associated software recorded the maximum force 
Table 1 Materials name, compositions, manufacturer, and application protocols

\begin{tabular}{|c|c|c|}
\hline Materials name & Chemical composition & Application protocols \\
\hline $\begin{array}{l}\text { Single Bond universal } \\
\text { adhesive } \\
\text { [3M ESPE, Deutschland, } \\
\text { GmbH, Germany; } \\
\text { Lot number: 5290463] }\end{array}$ & $\begin{array}{l}\text {-MDP phosphate mono- } \\
\text { mer, dimethacrylate resins, } \\
\text { Vitrebond copolymer, HEMA, } \\
\text { filler, ethanol, water, initiators, } \\
\text { silane } \mathrm{pH}=2.7\end{array}$ & $\begin{array}{l}\text { Self-etch adhesion protocol: Single Bond universal adhesive was applied to } \\
\text { the assigned dentin area for bonding using a microbrush and rubbed for } 20 \\
\text { seconds. The adhesive layer was air-thinned using gentle oil-free compressed } \\
\text { air for } 5 \text { seconds to evaporate the solvent. The adhesive layer was light cured } \\
\text { for } 10 \text { seconds according to the manufacturer's instruction using LED light } \\
\text { curing unit at intensity } 1,000 \mathrm{~mW} / \mathrm{cm}^{2} \\
\text { Etch-and-rinse adhesion protocol: Specimens assigned for this group were } \\
\text { first acid etched with } 37 \% \text { phosphoric acid gel [Meta Biomed, Germany. Lot } \\
\text { number: MET1906071] for } 15 \text { seconds followed by rinsing thoroughly with } \\
\text { water for } 15 \text { seconds. Specimens were then blot dried. Single Bond universal } \\
\text { adhesive was then applied to the assigned dentin area for bonding as men- } \\
\text { tioned in the self-etch adhesion protocol }\end{array}$ \\
\hline $\begin{array}{l}\text { Prime\&Bond universal } \\
\text { adhesive } \\
\text { [Dentsply DeTrey } \\
\text { GmbH, Germany. Lot } \\
\text { number 1811000038] }\end{array}$ & $\begin{array}{l}\text {-Phosphoric acid modified } \\
\text { acrylate resin, bi- and mul- } \\
\text { tifunctional acrylate, acidic } \\
\text { monomers (PENTA and MDP), } \\
\text { isopropanol, water, initiator, } \\
\text { stabilizer, crosslinking (N-ally), } \\
\text { pH > } 2.5\end{array}$ & $\begin{array}{l}\text { Self-etch adhesion protocol: Prime\&Bond universal adhesive was applied to } \\
\text { the assigned dentin area for bonding and agitated for } 20 \text { seconds. The adhe- } \\
\text { sive layer was air-thinned using gentle oil-free compressed air for } 5 \text { seconds } \\
\text { to evaporate the solvent. The adhesive layer was light cured for } 10 \text { seconds } \\
\text { according to the manufacturer's instruction using LED light curing unit at } \\
\text { intensity } 1,000 \mathrm{~mW} / \mathrm{cm}^{2} \\
\text { Etch-and-rinse adhesion protocol: Specimens assigned for this group were } \\
\text { acid etched with } 37 \% \text { phosphoric acid gel for } 15 \text { seconds followed by rinsing } \\
\text { thoroughly with water for } 15 \text { seconds. Specimens were then plot dried to } \\
\text { remove excess water using cotton pellets. Then Prime\&Bond universal adhe- } \\
\text { sive was applied to the assigned dentin area for bonding as mentioned in the } \\
\text { self-etch adhesion protocol }\end{array}$ \\
\hline
\end{tabular}

Abbreviations: HEMA, hydroxyethymethacrylate; MDP, methacryloyloxydecyl dihydrogen phosphate; PENTA, dipentaerythritol penta-acrylate phosphate.

needed to debond each specimen in Newton $(\mathrm{N})$, which automatically calculated it to express the SBS records on output device in MPa. ${ }^{9}$

\section{Mode of Failure Analysis}

The fractured test specimens from the SBS testing were assessed by stereomicroscope (50×) (Nikon SMZ-2, Japan) and the mode of failure (adhesive, cohesive, or mixed) was determined. The failure mode was categorized according to the area of resin remained on the dentin surface as follow ${ }^{10}$ :

Adhesive failure: resin composite remained at the interfacial bond area not exceed $25 \%$.

Cohesive failure: resin composite remained at the interfacial bond area equal to or greater than $75 \%$.

Mixed adhesive/cohesive failure: resin composite remained at the interfacial bond area was 25 to $75 \%$.

\section{Statistical Analysis}

The SBS mean and standard deviation (SD) values were calculated for each group. Data were explored for normality using Kolmogorov-Smirnov and Shapiro-Wilk tests, data showed parametric (normal) distribution. Independent sample $t$-test was used to compare between two groups in nonrelated specimens. Three-way analysis of variance (ANOVA) tests were used to test the interactions between different variables. The significance level was set at $p \leq 0.05$. Statistical analysis was performed with IBM SPSS Statistics Version 20 for Windows.

\section{Results}

\section{Results of Shear Bond Strength Measurements}

Results of three-way ANOVA for the effect of dentin substrate, universal adhesive, and adhesion protocol as independent variables and their interaction on the SBS (MPa) are presented in - Table 2. Mean and SD of the SBS values for different dentin substrates (A) bonded with Single Bond universal adhesive $\left(B_{1}\right)$ and Prime\&Bond universal with different adhesion protocols $(\mathrm{C})$ are shown in - Table $\mathbf{3}$. Mean and SD of the SBS values of groups bonded with different universal adhesive systems to sound dentin and CAD using etch-and-rinse and self-etch adhesion protocols are shown in - Table 4. On the other hand, mean and SD of SBS values of the different adhesion protocols applied to sound dentin and CAD when bonded with different universal adhesives are shown in - Table 5.

\section{Results of Failure Mode Analysis}

Percentage mode of failure of Single Bond universal adhesive systems $\left(B_{1}\right)$ and Prime\&Bond universal (B2) applied with different adhesion protocols $(C)$ bonded to different dentin substrates (A) are presented in -Figs. 1 and 2, respectively.

\section{Discussion}

As the bonded substrate had a great influence on the restoration success, both sound and CAD were tested in this study. Generation of artificial CAD was selected for this study rather than collection of natural CAD specimens. Despite of the some morphological differences between natural and artificially created CAD (e.g., tubular occlusion with whitlockite minerals and microhardness) the bond strengths do not seem to be affected by the type of caries, allowing the use of laboratory models that overcome the huge inconsistency of natural CAD. ${ }^{11,12}$ Artificially created CAD method allowed standardization of the degree of dentin demineralization across all tested specimens. In addition, the method selected 


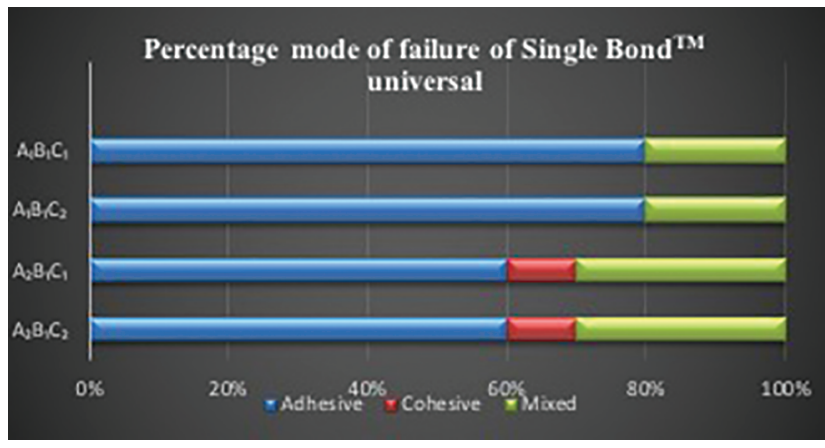

Fig. 1 Percentage mode of failure of Single Bond universal adhesive systems $\left(\left[A_{1} B_{1} C_{1}\right]\right.$ : Single Bond universal bonded to sound dentin with etch-and-rinse; $\left[A_{1} B_{1} C_{2}\right]$ : Single Bond universal bonded to sound dentin self-etch adhesion protocol; $\left[A_{2} B_{1} C_{1}\right]$ : Single Bond universal bonded to caries-affected dentin with etch-and-rinse adhesion protocol; $\left[A_{2} B_{1} C_{2}\right]$ : Single Bond universal bonded to caries-affected dentin with self-etch adhesion protocol).

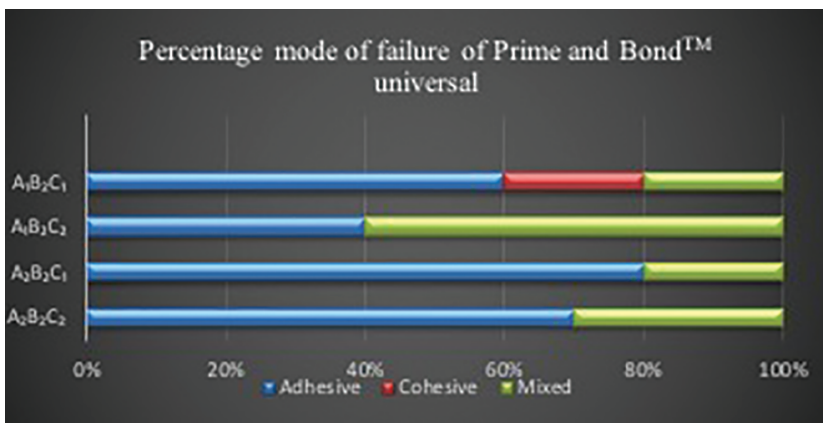

Fig. 2 Percentage mode of failure of Prime\&Bond universal adhesive systems $\left(\left[A_{1} B_{2} C_{1}\right]\right.$ : Prime\&Bond universal bonded to sound dentin with etch-and-rinse; $\left[A_{1} B_{2} C_{2}\right]$ : Prime\&Bond universal bonded to sound dentin self-etch adhesion protocol; $\left[\mathrm{A}_{2} \mathrm{~B}_{2} \mathrm{C}_{1}\right]$ : Prime\&Bond universal bonded to caries-affected dentin with etch-and-rinse adhesion protocol; $\left[\mathrm{A}_{2} \mathrm{~B}_{2} \mathrm{C}_{2}\right]$ : Prime\&Bond universal bonded to caries-affected dentin with self-etch adhesion protocol).

Table 2 Results of three-way ANOVA for the effect of different variables on shear bond strength

\begin{tabular}{|l|l|l|l|l|l|}
\hline Source & $\begin{array}{l}\text { Type III sum of } \\
\text { squares }\end{array}$ & df & Mean square & $\boldsymbol{F}$ & Significance \\
\hline Corrected model & 207.107 & 7 & 29.587 & 5.852 & $0.000^{\text {a }}$ \\
\hline Intercept & $5,001.446$ & 1 & $5,001.446$ & 989.199 & $0.000^{\text {a }}$ \\
\hline Dentin substrate & 92.474 & 1 & 92.474 & 18.290 & $0.000^{\text {a }}$ \\
\hline Multimode adhesive & 16.284 & 1 & 16.284 & 3.221 & 0.077 \\
\hline Adhesion protocols & 29.339 & 1 & 29.339 & 5.803 & $0.019^{\text {a }}$ \\
\hline Dentin substrate * Multimode adhesive & 31.487 & 1 & 31.487 & 6.228 & $0.015^{\text {a }}$ \\
\hline Dentin substrate * Adhesion protocols & 26.119 & 1 & 26.119 & 5.166 & $0.026^{\text {a }}$ \\
\hline Multimode adhesive * Adhesion protocols & 11.365 & 1 & 11.365 & 2.248 & 0.138 \\
\hline $\begin{array}{l}\text { Dentin substrate * Multimode adhesive * } \\
\text { Adhesion protocols }\end{array}$ & 0.040 & 1 & 0.040 & 0.008 & 0.929 \\
\hline Error & 364.036 & 72 & 5.056 & & \\
\hline Total & $5,572.589$ & 80 & & & \\
\hline Corrected total & 571.143 & 79 & & & \\
\hline
\end{tabular}

Abbreviations: ANOVA, analysis of variance; $\mathrm{df}$, degrees of freedom $(n-1)$.

aSignificant at $p \leq 0.05$.

Table 3 The mean and standard deviation (SD) values of shear bond strength of different dentin substrate groups

\begin{tabular}{|c|c|c|c|c|c|c|c|c|}
\hline \multirow[t]{4}{*}{ Variables } & \multicolumn{8}{|c|}{ Shear bond strength } \\
\hline & \multicolumn{4}{|c|}{ Single Bond universal $\left(B_{1}\right)$} & \multicolumn{4}{|c|}{ Prime \& Bond universal $\left(\mathrm{B}_{2}\right)$} \\
\hline & \multicolumn{2}{|c|}{ Etch-and-rinse protocol $\left(C_{1}\right)$} & \multicolumn{2}{|c|}{$\begin{array}{l}\text { Self-etch } \\
\text { protocol }\left(C_{2}\right)\end{array}$} & \multicolumn{2}{|c|}{$\begin{array}{l}\text { Etch-and-rinse } \\
\text { protocol }\left(C_{1}\right)\end{array}$} & \multicolumn{2}{|c|}{ Self-etch protocol $\left(C_{2}\right)$} \\
\hline & Mean & SD & Mean & SD & Mean & SD & Mean & SD \\
\hline $\begin{array}{l}\text { Sound dentin } \\
\left(A_{1}\right)\end{array}$ & 11.59 & 2.48 & 8.53 & 1.96 & 8.73 & 1.77 & 7.08 & 2.41 \\
\hline $\begin{array}{l}\text { Caries-affected } \\
\text { dentin }\left(A_{2}\right)\end{array}$ & 7.09 & 2.32 & 6.22 & 2.56 & 6.64 & 2.61 & 7.37 & 1.64 \\
\hline$p$-Value & \multicolumn{2}{|l|}{$0.001^{\mathrm{a}}$} & \multicolumn{2}{|l|}{$0.036^{a}$} & \multicolumn{2}{|c|}{0.052 (ns) } & \multicolumn{2}{|c|}{0.755 (ns) } \\
\hline
\end{tabular}

aSignificant $(p<0.05)$, nonsignificant $(\mathrm{ns})(p>0.05)$.

for development of CAD in this study was claimed to produce effective depth of demineralized dentin to more than $100 \mu \mathrm{m} .{ }^{11}$
Regarding the results of the effect of the tooth substrate either, sound or CAD on the SBS (MPa) values for the tested universal adhesive systems irrespective to other variables of 
Table 4 The mean and standard deviation (SD) values of shear bond strength of different multimode adhesive groups

\begin{tabular}{|c|c|c|c|c|c|c|c|c|}
\hline \multirow[t]{4}{*}{ Variables } & \multicolumn{8}{|c|}{ Shear bond strength } \\
\hline & \multicolumn{4}{|c|}{ Sound dentin $\left(A_{1}\right)$} & \multicolumn{4}{|c|}{ Caries-affected dentin (CAD) $\left(A_{2}\right)$} \\
\hline & \multicolumn{2}{|c|}{ Etch-and-rinse protocol $\left(C_{1}\right)$} & \multicolumn{2}{|c|}{$\begin{array}{l}\text { Self-etch } \\
\text { protocol }\left(C_{2}\right)\end{array}$} & \multicolumn{2}{|c|}{$\begin{array}{l}\text { Etch-and-rinse } \\
\text { protocol }\left(C_{1}\right)\end{array}$} & \multicolumn{2}{|c|}{$\begin{array}{l}\text { Self-etch protocol } \\
\left(C_{2}\right)\end{array}$} \\
\hline & Mean & SD & Mean & SD & Mean & SD & Mean & SD \\
\hline $\begin{array}{l}\text { Single Bond univer- } \\
\text { sal }\left(B_{1}\right)\end{array}$ & 11.59 & 2.48 & 8.53 & 1.96 & 7.09 & 2.32 & 6.22 & 2.56 \\
\hline $\begin{array}{l}\text { Prime\&Bond uni- } \\
\text { versal }\left(B_{2}\right)\end{array}$ & 8.73 & 1.77 & 7.08 & 2.41 & 6.64 & 2.61 & 7.37 & 1.64 \\
\hline$p$-Value & \multicolumn{2}{|l|}{$0.008^{\mathrm{a}}$} & \multicolumn{2}{|c|}{0.158 (ns) } & \multicolumn{2}{|c|}{0.691 (ns) } & \multicolumn{2}{|c|}{0.247 (ns) } \\
\hline
\end{tabular}

aSignificant $(p<0.05)$, nonsignificant (ns) $(p>0.05)$.

Table 5 The mean and standard deviation (SD) values of shear bond strength of different adhesion protocols groups

\begin{tabular}{|c|c|c|c|c|c|c|c|c|}
\hline \multirow[t]{4}{*}{ Variables } & \multicolumn{8}{|c|}{ Shear bond strength } \\
\hline & \multicolumn{4}{|c|}{ Sound dentin $\left(A_{1}\right)$} & \multicolumn{4}{|c|}{ Caries-affected dentin (CAD) $\left(A_{2}\right)$} \\
\hline & \multicolumn{2}{|c|}{ Single Bond universal $\left(B_{1}\right)$} & \multicolumn{2}{|c|}{$\begin{array}{l}\text { Prime\&Bond } \\
\text { universal }\left(B_{2}\right)\end{array}$} & \multicolumn{2}{|c|}{$\begin{array}{l}\text { Single Bond } \\
\text { universal }\left(B_{1}\right)\end{array}$} & \multicolumn{2}{|c|}{$\begin{array}{l}\text { Prime\&Bond universal } \\
\left(B_{2}\right)\end{array}$} \\
\hline & Mean & SD & Mean & SD & Mean & SD & Mean & SD \\
\hline $\begin{array}{l}\text { Etch-and-rinse } \\
\text { protocol }\left(C_{1}\right)\end{array}$ & 11.59 & 2.48 & 8.73 & 1.77 & 7.09 & 2.32 & 6.64 & 2.61 \\
\hline $\begin{array}{l}\text { Self-etch pro- } \\
\text { tocol }\left(C_{2}\right)\end{array}$ & 8.53 & 1.96 & 7.08 & 2.41 & 6.22 & 2.56 & 7.37 & 1.64 \\
\hline$p$-Value & \multicolumn{2}{|l|}{$0.007^{\mathrm{a}}$} & \multicolumn{2}{|c|}{0.099 (ns) } & \multicolumn{2}{|c|}{0.438 (ns) } & \multicolumn{2}{|c|}{0.464 (ns) } \\
\hline
\end{tabular}

aSignificant $(p<0.05)$, nonsignificant (ns) $(p>0.05)$.

the study and results of SBS (MPa) values for the effect of different dentin substrates bonded with Single Bond universal with different adhesion protocols (-Table $\mathbf{3}$ ), showed that sound dentin had statistically significant higher SBS (MPa) values than CAD. These findings were in agreement with previous studies. ${ }^{6,7,13,14}$

The possible explanation for the lower bond strength of CAD in comparison to sound dentin were that CAD lost a part of its mineral composition from the intertubular dentin during the demineralization process. The volume of the lost minerals from the CAD matrix was replaced by water. This increase in the water content and the more permeable condition were considered factors that might compromise the bonding quality. In addition, other chemical and morphological alteration that occur in the demineralized CAD, such as loss of its organic content, the collagen fibrils with lower collagen cross-linkages, increased porosity of intertubular dentin, dissolution of apatite crystals, and degradation of collagen fibrils, were other factors that might negatively affect bonding to that substrate. Moreover, the matrix of the peritubular dentin in CAD contain noncollagenous protein content (mucopolysaccharide or glycoprotein molecules) which could interfere with the adhesion. ${ }^{6,14,15}$ All the previously stated microstructural changes in CAD might negatively impact the performance of the dental adhesives.

In addition, the lower mineral content of CAD allowed phosphoric acid or acidic monomers to demineralize the matrix more deeply than normal dentin, resulting in even more residual water in exposed collagen matrix. Therefore, the formed hybrid layer was expected to be thicker and more porous compared with the hybrid layer formed on sound dentin due to the demineralization process, which negatively influence the bond strength. ${ }^{7}$

On the other hand, results of Prime\&Bond universal adhesive revealed a nonstatistically significant SBS (MPa) values between bonding to sound and CAD (- Table 3 ). This might be due to the unique chemical composition of Prime\&Bond universal adhesive. It contained a newly developed hydrolysis stable cross-linker ( $\mathrm{N}$-ally functions). As stated by the manufacturer, this universal adhesive was designed to retain all the benefits of a standard acryl resin adhesive, but also added water compatibility within one single monomer. The result was a new liquid, bifunctional acryl cross-linker, containing $\mathrm{N}$-ally functions to achieve a high level of polymerization reactivity. This adhesive achieved an optimized surface tension and a low contact angle on dental substrates, thus avoiding phase separation when bonding to more water contained substrate as in cases of the porous, water-filled CAD.

Regarding the results of the effect of the of different universal adhesives on mean SBS (MPa), there was nonstatistically significant difference between Single Bond universal adhesive and Prime\&Bond universal adhesive. This might be due to the chemical composition of both tested universal adhesives. Both universal adhesives were mild adhesives with $\mathrm{pH}$ of 2.7 for Single Bond universal adhesive and $\mathrm{pH}>$ 2.5 for Prime\&Bond universal adhesive.

Moreover, both universal adhesives contained 10-methacryloyloxydecyl dihydrogen phosphate monomer (10-MDP), 
which is a phosphate monomer that gives the adhesive an acidic character, enabling simultaneous demineralization and monomer infiltration. In addition, 10-MDP established a chemical bond to dentin via its phosphate group, which bind ionically to residual calcium attached to the collagen fibrils forming a stable and water-resistant Ca-monomer salt created by the reaction of 10-MDP and the $\mathrm{Ca}^{+}$ions of hydroxyapatite. ${ }^{16,17}$

The only exception was shown when the universal adhesive systems were applied to sound dentin with etch-and-rinse adhesion protocol (-Table 4) where Single Bond universal had higher statistically significant SBS mean value compared with Prime\&Bond universal.

Sound dentin is formed of nearly $50 \%$ inorganic apatite crystals, $30 \%$ collagen, and $20 \%$ water by volume. In the etch-and-rinse adhesion protocol, the surface and subsurface minerals were solubilized, removed, and were substituted by rinse-water, which combined with intrinsic water, surrounding the collagen fibrils. ${ }^{18}$

Single Bond universal superiority on the etched sound dentin substrate might be due to its unique chemistry that optimizes the ratios of proprietary Vitrebond copolymer, 2-hydroxyethylmethacrylate (HEMA), and ethanol/water solvent. The affinity of HEMA in Single Bond universal seems to be enhanced when it is combined with water displacing solvents like ethanol. This adhesive formula allowed a decrease in its thickness and viscosity that eases wetting of the etched dentin surface and keeps the collagen fibrils in an expanded form after the evaporation of solvents, enhancing monomers penetration into wet and dry dentin substrates. This result in effective formation of a resin-demineralized dentin hybrid layer, that result in a strong micromechanical interlocking between resin and the superficially demineralized tooth substrate. ${ }^{18,19}$

Moreover, Single Bond universal contained polyalkenoic acid copolymer (Vitrebond copolymer). This polyalkenoic acid copolymer according to the manufacturer provided satisfactory bonding to wet and dry dentin. Moreover, it could form Ca-polyalkenoate complexes at the superficial zone of the hybrid layer and within the superficial $3 \mu \mathrm{m}$ of dentinal tubules, which could stabilize the bonded interface by offering water stability and a stress-relaxing outcome. ${ }^{18,19}$

The current results indicated that the capability of the universal adhesives in bonding to dentin, considering the adhesion protocol was material-dependent. This denotes that the composition of the materials tested, comprising type and the amount of acidic monomers, hydrophobic and hydrophilic methacrylates, photoinitiators, solvents, and other constitutions, might influence their bonding performance. ${ }^{19,20}$

A recent systematic review of in vitro studies conducted by Rosa et $\mathrm{al}^{21}$ stated that enamel bond strength of universal adhesives was enhanced with prior etching using phosphoric acid, but the same result was not obvious for dentin when mild universal adhesives were utilized. Same results were observed in the current study, since no differences in bond strength were detected between the bonding protocols (etch-and-rinse or self-etch) when bonded to sound and CAD. The only exception was Single Bond universal, that had a statistically higher resin-dentin bond strength when used in the etch-and-rinse mode on sound dentin compared with the self-etch adhesion protocol.

In previous studies, they found that the etching step improves the penetration of the adhesive on sound dentin, resulting in longer resin tags and thicker hybrid layers. ${ }^{17,19,20}$ In contrast, different results were observed in the literature $^{6,10,22}$ in which some authors found no differences in bond strength of Single Bond universal when applied to sound dentin, regardless of the strategies used. This contradiction in the results might be due to difference in methodology in which the adhesive system was tested under different conditions.

Failure mode analysis was considered a significant parameter for understanding the testing results when measuring the bond strength between two materials. It has been reported that there was as direct positive correlation between the bond strength and the mode of failure. ${ }^{7,15}$ On the other hand, others found that there was no direct relationship between the fracture mode and the SBS. In this study, three modes of failures were observed which were adhesive, cohesive, or mixed. The adhesive and the mixed types of failure were the more prominent types with both universal adhesive in the different groups, which was considered to be a true indication for the bond effectiveness of these universal adhesives to different dentin substrates. In contrast to the cohesive mode of failure which was considered to be indicative of higher resin-dentin bond strength. . $^{63}$

Finally, in the present study the first null hypothesis that there was no difference in resin-dentin SBS of CAD and sound dentin was partially rejected as there was statistically significant difference between the bonded substrates using Single Bond universal.

Moreover, the second null hypothesis was also partially rejected, as there was no difference in resin-dentin SBS between the two tested universal adhesive systems except when they were bonded to sound dentin in the etch-and-rinse adhesion protocol.

While regarding the third hypothesis that there was no difference in resin-dentin SBS between the different adhesion protocols (either in etch-and-rinse or self-etch), it was totally accepted for Prime\&Bond universal adhesive, while partially rejected for Single Bond universal, as there was difference between the adhesion protocols when bonded to sound dentin only.

\section{Conclusion}

The influence of CAD on the performance of the universal adhesives was material-dependent, as it only jeopardized bonding of Single Bond universal adhesive. Both tested universal adhesive systems had comparable performance on different dentin substrates, except when they were bond to sound dentin with etch-and-rinse protocol, where Single Bond universal adhesive performed better. The effect of different adhesion protocols on the performance of the universal adhesives was material-dependent, as application of Single Bond universal adhesive in an etch-and-rinse adhesion 
protocol improved only bonding to sound dentin, while no added positive effect for the etching step with Prime\&Bond universal adhesive when bonded to both sound dentin and CAD.

\section{Recommendations}

1. It is recommended to use Single Bond universal with etch-and-rinse adhesion protocol when bonding to sound dentin.

2. Using universal adhesive systems in self-etch adhesion protocol when bonding to caries-affected dentin.

\section{Funding}

This research did not receive any specific grant from funding agencies in the public, commercial, or not-for-profit sectors.

\section{Note}

The manuscript has been read and approved by all the authors, that the requirements for authorship have been met, and each author believes that the manuscript represents honest work.

\section{Conflict of Interest}

The authors have declared that no conflicts of interest exist.

\section{References}

1 Costa AR, Garcia-Godoy F, Correr-Sobrinho L, et al. Influence of different dentin substrate (caries-affected, caries-infected, sound) on long-term $\mu$ TBS. Braz Dent J 2017;28(1):16-23

2 Yoshiyama M, Tay FR, Doi J, et al. Bonding of self-etch and total-etch adhesives to carious dentin. J Dent Res 2002;81(8):556-560

3 Sofan E, Sofan A, Palaia G, Tenore G, Romeo U, Migliau G. Classification review of dental adhesive systems: from the IV generation to the universal type. Ann Stomatol (Roma) 2017;8(1):1-17

4 Shibata S, Vieira LCC, Baratieri LN, et al. Evaluation of microtensile bond strength of self-etching adhesives on normal and caries-affected dentin. Dent Mater J 2016;35(2):166-173

5 Nagi SM. Durability of solvent-free one-step self-etch adhesive under simulated intrapulpal pressure. J Clin Exp Dent 2015;7(4):e466-e470

6 Nicoloso GF, Antoniazzi BF, Lenzi TL, Soares FZM, Rocha RO. The bonding performance of a universal adhesive to artificially-created caries-affected dentin. J Adhes Dent 2017;19(4):317-321

7 Shadman N, Farzin-Ebrahimi S, Mortazavi-Lahijani E, Ghaderi A. Shear bond strength of different adhesive systems to normal and caries-affected dentin. Journal OHOE 2015;4:87-93
8 Pashley DH, Tay FR, Yiu C, et al. Collagen degradation by host-derived enzymes during aging. J Dent Res 2004;83(3):216-221

9 Jayasheel A, Niranjan N, Pamidi H, Suryakanth MB. Comparative evaluation of shear bond strength of universal dental adhesives. J Clin. Exp 2017;9:892-896

10 Isolan CP, Valente LL, Münchow EA, et al. Bond strength of a universal bonding agent and other contemporary dental adhesives applied on enamel, dentin, composite, and porcelain. Appl Adhes Sci 2014;2:25-35

11 Follak AC, Miotti LL, Lenzi TL, Rocha RO, Soares FZM. Influence of water storage on bond strength of multimode adhesive systems to artificially induced caries-affected dentin. Oper Dent 2018;43:92-101

12 Joves GJ, Inoue G, Nakashima S, Sadr A, Nikaido T, Tagami J. Mineral density, morphology and bond strength of natural versus artificial caries-affected dentin. Dent Mater J 2013;32(1):138-143

13 Drobac M, Stojanac I, Ramić B, Premović M, Petrović L. Shear bond strength to sound and caries-affected dentin of simplified "etch-and-rinse" and "self-etch" adhesives and the hybrid layer micromorphology. Vojnosanit Pregl 2019;76:675-683

14 Follak AC, Miotti LL, Lenzi TL. Rocha RO, Maxnuck Soares FZ. The impact of artificially caries-affected dentin on bond strength of multi-mode adhesives. J Conserv Dent 2018;21(2):136-141

15 Müller C, Teixeira GS, Krejci I, Bortolotto T, Susin AH. Effect of caries-affected dentin on one-step universal and multi-step etch-and-rinse adhesives' bond strength. Rev Odontol UNESP 2017;46:273-277

16 Ghajari MF, Ghasemi A, Badiee M, Abdolazimi Z, Baghban AA. Microshear bond strength of scotchbond universal adhesive to primary and permanent dentin. Front Dent 2019;16(3):173-180

17 Yamauchi K, Tsujimoto A, Jurado CA, et al. Etch-and-rinse vs self-etch mode for dentin bonding effectiveness of universal adhesives. J Oral Sci 2019;61(4):549-553

18 Goud KM, Arun J, Nishanth P, Deepak BS, Nandini TN. Comparative evaluation of shear bond strength of three dental adhesives under dry and wet bonding conditions. J Int Oral Health 2016;8:267-271

19 Ahmed AA, Hassan MM, Abdalla AI. Microshear bond strength of universal adhesives to dentin used in total-etch and self-etch modes. Tanta Dent. J 2019;15:91-98

20 Montagner AF, Carvalho MP, Susin AH. Microshear bonding effectiveness of different dentin regions. Indian J Dent Res 2015;26(2):131-135

21 Rosa WL, Piva E, Silva AF. Bond strength of universal adhesives: a systematic review and meta-analysis. J Dent 2015;43(7):765-776

22 Hanabusa M, Mine A, Kuboki T, et al. Bonding effectiveness of a new 'multi-mode' adhesive to enamel and dentine. J Dent 2012;40(6):475-484

23 Memarpour M, Shafiei F, Razmjouei F, Soltani M. Shear bond strength and scanning electron microscopy characteristics of universal adhesive in primary tooth dentin: an in vitro study. Dent Res J (Isfahan) 2018;15(4):264-270 